\title{
Dermoscopy in the COVID-19 Era: Magnifying the Gap for Clinicians
}

\author{
Khawar Hussain ${ }^{1}$, Ashfaq A. Marghoob ${ }^{2}$, Neil P. Patel ${ }^{1}$
}

1 Department of Dermatology, Charing Cross Hospital, Imperial College Healthcare NHS Trust, London, UK

2 Department of Dermatology, Memorial Sloan Kettering Cancer Center, New York, USA

Key words: dermoscopy, COVID-19, medical education, skin cancer, teledermatology

Citation: Hussain K, Marghoob AA, Patel NP. Dermoscopy in the COVID-19 era: magnifying the gap for clinicians. Dermatol Pract Concept. 2021;11(2):e2021069. DOI: https://doi.org/10.5826/dpc.1102a69

Accepted: February 1, 2021; Published: March 8, 2021

Copyright: $@ 2021$ Hussain et al. This is an open-access article distributed under the terms of the Creative Commons Attribution License BY-NC-4.0, which permits unrestricted noncommercial use, distribution, and reproduction in any medium, provided the original author and source are credited.

Funding: None.

Competing interests: The authors have no conflicts of interest to disclose.

Authorship: All authors have contributed significantly to this publication.

Corresponding author: Dr. Khawar Hussain, Charing Cross Hospital, Imperial College Healthcare NHS Trust, Fulham Palace Road London W6 8RF, UK. Email: khawar.hussain1@nhs.net

Dermoscopy is an invaluable tool that has improved the diagnostic ability of clinicians to differentiate between benign and malignant skin lesions by up to $25 \%$ [1]. It is practiced using a handheld device and interpretation is operator-dependent, such that expertise in this technique requires many hours of training. Its greatest use is in the diagnosis of pigmented lesions, but dermoscopy also has an important role in the diagnosis of nonmelanoma skin cancers, benign lesions, inflammatory disorders, and even parasitic infections.

The advent of teledermatology, hastened by the ongoing COVID-19 pandemic, has changed the dynamic of the doctor-patient consultation and has led to extensive replacement of face-to-face consultations with virtual consultations. For teledermatological assessment of patients with suspected skin cancer, the historic practice of performing a face-to-face full skin examination has been supplanted by macroscopic and dermoscopic photographs of a specific skin lesion. Traditionally, clinicians have been able to intuitively gather important diagnostic clues from a dynamic dermoscopic examination of skin lesions and have taken this method of examination for granted. As teledermatology generates static dermoscopic images and omits these dynamic dermoscopic clues, we are only now beginning to realize what has been lost.

Such clues and techniques include the wobble sign for intradermal nevi [2], ink test for seborrheic keratosis [3] and porokeratosis [4], furrow ink test for pigmented acral lesions [5], oblique view dermoscopy for fibrillar-pattern pigmented lesions on the sole [6], and tape stripping of hypermelanotic nevi [7]. These dynamic dermoscopic tricks augment the established dynamic clinical tests practiced for various skin lesions during face-to-face consultations, including the pinch test for dermatofibroma, the scratch test for subcorneal hematoma [8], and lifting of crusts to examine hyperkeratotic lesions.

As the field of teledermoscopy evolves, it is important that dermatologists maximize the full potential of this technique, which would include embedding dynamic dermoscopic clues into standard teledermoscopy practice. In the virtual era, this could be achieved by training medical photographers to elicit these clues; for example, the furrow ink test and accompanying 
dermoscopic photographs could be recommended as standard practice when photographing any pigmented lesion on the palm or sole. Potential drawbacks include the additional resource required for photographer training and the extra time needed per patient.

Additionally, where a supplementary dynamic clinical test of a skin lesion is deemed essential by the teledermatologist, such as the scratch test for a subcorneal hematoma, we should not be discouraged from asking patients to return to a conventional face-to-face dermatological consultation in this virtual era, as the stakes of a missed skin cancer diagnosis, as ever, are high.

Furthermore, despite the continuing shift towards virtual work, it is essential that we continue to provide dermatology trainees with teaching in techniques that can only be performed face-to-face, such as handheld dermoscopy, dynamic assessment of skin lesions, and full skin examinations. Neglecting these skills would regrettably lead to a new generation of dermatologists ill-equipped to conduct a thorough face-to-face examination of skin lesions.

\section{References}

1. Wolner ZJ, Yelamos O, Liopyris K, et al. Enhancing skin cancer diagnosis with dermoscopy. Dermatol Clin. 2017;35:417-437. DOI: 10.1016/j.det.2017.06.003. PMID: 28886798.
2. Braun RP, Krischer J, Saurat JH. The "wobble sign" in epiluminescence microscopy as a novel clue to the differential diagnosis of pigmented skin lesions. Arch Dermatol. 2000;136(7):940-942. DOI: 10.1001/archderm.136.7.940-a. PMID: 10891009.

3. Yagerman S, Marghoob AA. The ink test: identifying 3-dimensional features of seborrheic keratoses under dermoscopy. JAMA Dermatol. 2013;149(4):497-498. DOI: 10.1001/jamadermatol.2013.2233. PMID: 23715284.

4. Navarrete-Dechent C, Uribe P, Marghoob A. Ink-enhanced dermoscopy is a useful tool to differentiate acquired solitary plaque porokeratosis from other scaly lesions. J Am Acad Dermatol. 2019;80(6):e137-e138. DOI: 10.1016/j.jaad.2017.11.052. PMID: 29221722.

5. Braun RP, Thomas L, Kolm I, French LE, Marghoob AA. The furrow ink test: a clue for the dermoscopic diagnosis of acral melanoma vs nevus. Arch Dermatol. 2008;144(12):1618-1620. DOI: 10.1001/archderm.144.12.1618. PMID: 19075144.

6. Maumi Y, Kimoto M, Kobayashi K, Ito N, Saida T, Tanaka M. Oblique view dermoscopy changes regular fibrillar pattern into parallel furrow pattern. Dermatology. 2009;218(4):385-386. DOI: 10.1159/000202986. PMID: 19218789.

7. Babino G, Specchio F, Lallas A, Longo C, Moscarella E, Argenziano G. Tape stripping: A very short-term follow-up procedure for suspicious black lesions. J Am Acad Dermatol. 2015;72(6):e151-e152. DOI: 10.1016/j.jaad.2015.02.1098. PMID: 25981018.

8. Zalaudek I, Argenziano G, Soyer HP, Saurat JH, Braun RP. Dermoscopy of subcorneal hematoma. Dermatol Surg. 2004;30(9):12291232. DOI: 10.1111/j.1524-4725.2004.30381.x. PMID: 15355366 . 